\title{
COMBATing Breast Cancer Conference 2012 - Deciphering Breast Cancer
}

\author{
Lisa Richters $^{\mathrm{a}, \mathrm{b}} \quad$ Rachel Wuerstlein $^{c} \quad$ Julian Puppe ${ }^{\mathrm{a}, \mathrm{b}}$ \\ aDepartment of Obstetrics and Gynecology, Breast Center, University of Cologne, \\ ${ }^{b} \mathrm{ClO}$ Cologne-Bonn, Cologne, \\ 'Department of Obstetrics and Gynecology, Breast Center, LMU Munich, Germany
}

The 5th COMBATing (Conference On Molecular Basics And Therapeutic implications in breast cancer) meeting was held in Munich, November 23-24, 2012. Breast cancer is a heterogeneous disease on the molecular, histopathological, and clinical level. For this reason, this year's conference was dedicated to a better understanding of the diversity of breast cancer. Chaired by Nadia Harbeck (Munich), Achim Rody (Lübeck), and Michael Gnant (Vienna), COMBATing 2012 attracted over 260 physicians and medical scientists including national and international pioneers in breast cancer research. In two days, recent controversial aspects of translational breast cancer research and treatment were discussed, from the evaluation of traditional histopathological classifications, to clinical trials to the role of the molecular subtypes.

\section{AGO TraFo Pre-Symposium for Translational Research}

After the successful first AGO TraFo (Arbeitsgruppe Gynäkologische Onkologie, Kommission Translationale Forschung) pre-symposium 2011, the 2012 conference was again preceded by an AGO TraFo meeting, chaired by Tanja Fehm (Tübingen). This meeting served as an ideal platform for young researchers to discuss their ongoing projects on translational breast cancer research with experts in the fields. To support young clinicians on their way to science, the first session gave insights into project planning, grant application, and presenting skills followed by a keynote lecture of Nikolas Hendrik Stoecklein (Düsseldorf) about the fascinating world of tumor cells and their interaction with their environment.

The award winners of the last AGO TraFo meeting shared their data during an oral presentation (Kirsten Kübler (Bonn), best lecture; Helen Schneck (Tübingen), best poster). After an overview over the German study groups and the international impact of the local research landscape, the pre-symposium finished with a poster presentation. Prizes for the best four posters were awarded to Anna Emde (Rehovot, Israel), Sophie Doisneau-Sixou (Munich/Toulouse), Julian Puppe (Cologne) and Rui Pedro Lousa das Neves (Düsseldorf).

\section{The COMBATing Meeting}

The meeting started with an update on recent developments since the last COMBATing in 2011. Anton Scharl (Amberg) predicted a paradigm shift from adjuvant to neoadjuvant chemotherapy as the standard concept in certain subtypes (triple negative, HER2 positive) of primary breast cancer thereby improving surgical outcome and allowing an early evaluation of therapy effectiveness. Furthermore, the question of the necessity of chemotherapy in nodal positive breast cancer, the role of Everolimus in bone health and the interaction of the tumor with its microenvironment were discussed.

Breast cancer treatment has experienced several changes during the past decades due to discovery of specific prognostic and predictive biomarkers. Therefore, the next session focused on the evidence-based use of biomarkers in the clinic. Hans Kreipe (Hannover) gave an overview over traditional biomarkers, such as hormone receptors and the role of the proliferation marker Ki-67 in chemotherapy response and prognosis after neoadjuvant therapy. Unfortunately, the exact cut-off value for Ki-67 has not yet been validated and thus still has to be evaluated in ongoing studies like the WSG-ADAPTtrial. In addition, the medical and scientific benefit of the new molecular tests (e.g. Onkotype DX ${ }^{\circledR}$, Endopredict ${ }^{\circledR}$ ) and their role in DCIS were discussed. Novel discoveries for the therapy of hereditary breast cancer were presented by Rita Schmutzler (Cologne). 2012 was a very successful year for the German Consortium of Hereditary Breast and Ovarian Cancer with the detection of new potential high risk genes, which are currently under evaluation.

\section{KARGER}

Fax +497614520714

Information@Karger.com

www.karger.com (c) 2013 S. Karger GmbH, Freiburg

Accessible online at:

www.karger.com/brc 


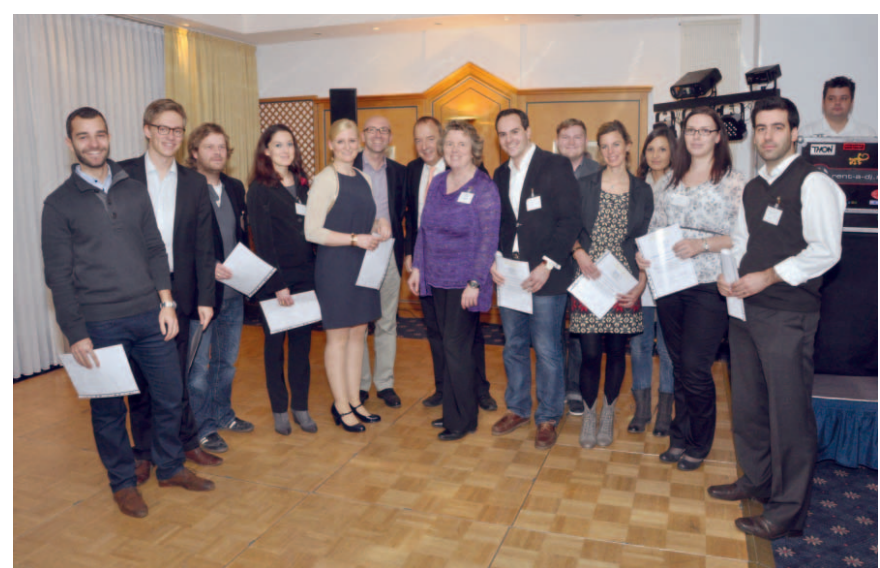

COMBAT Travel Grant winners were awarded by the three scientific chairs Achim Rody, Michael Gnant, and Nadia Harbeck.

From left to right: Thomas Kolben (Munich), Robert Armbrust (Munich), Christof Bernemann (Münster), Anna Emde (Rehovot, Israel), Hilke Göttsching (Lübeck), Achim Rody (Lübeck), Michael Gnant (Vienna), Nadia Harbeck (Munich), Alex Farr (Munich), Benjamin Leyh (Halle/ Saale), Katharina Beuster (Lübeck), Johanna Naskou (Düsseldorf), Katharina Cuk (Heidelberg), Rui Pedro Lousa das Neves (Düsseldorf).

In his keynote lecture, Stefan Glück (Miami, FL) gave an introduction into the rising significance of nanotechnology in breast cancer research. He highlighted the promising possibilities of nanotechnology by portraying the history of traditional paclitaxel to the highly evolved albumin-bound compound nab-paclitaxel, calling it the 'beginning of the future'. The clinical impact of nab-paclitaxel is currently tested in the Geparsepto and ICE II trials.

With the main focus on deciphering the increasing heterogeneity of breast cancer, the next session took the challenge to highlight its clinical implications. As discussed by Rachel Würstlein (Munich), the tumor diversity has a major impact on the clinical course of breast cancer and therapy response. This includes changes in tumor biology during breast cancer progression. Consequently, re-testing of metastases should be performed to re-assess tumor biology and thus improve the efficacy of therapy whenever accessible.

Next-generation sequencing technologies are able to characterize cancer at genomic, transcriptomic, and epigenetic levels. With these new techniques, we are able to get deeper insights into intratumoral heterogeneity by analyzing the whole tumor genome as explained by Thomas Karn (Frankfurt/M.). Peter Fasching (Erlangen) presented new therapeutic implications of whole-genome sequencing to predict therapy response. From a visionary point of view, these novel methods can be used to detect low and high proliferating tumor classes and to monitor the mutation status during tumor development. But next-generation sequencing will require high-performance bio-informatic support, quality control, and standardization, which would limit its clinical implementation to specialized centers. Overall, Prof. Fasching pointed out that without the clinicians' commitment, the translation from bench to bedside will never be possible. 'We need the clinicians!'

Whole-genome analysis has revolutionized breast cancer research with the identification of molecular subtypes. Thus, the following lectures were dedicated to the different molecular subtypes, their diagnostics and specialized treatments. As presented by Günther Steger (Vienna), the HER2-positive subtype will profit from new target-orientated therapies, such as the trastuzumab-emtansine conjugate T-DM1 and the HER2-dimerization inhibitor pertuzumab. Pertuzumab is already awaiting approval in Germany for the beginning of 2013 and T-DM1 will be available within several trials for (neo-) adjuvant and palliative treatment of HER2-overexpressing breast tumors.

With the implementation of targeted therapies, we have to deal with novel unexpected side effects. Here, Hans Tesch (Frankfurt/M.) discussed possible adverse events of mTORand PI3K-inhibitors and their clinical management. Focusing on restricted resources in the healthcare system, Michael Lux (Erlangen) finished the session with a discussion about the economic aspects of personalized breast cancer diagnostics and treatment. With a glimpse into the future, Timo Schinköthe (Munich) gave insights into the emerging role of eHealth with the use of smart phones as source of information and to improve doctor-patient communication.

With the 5th COMBATing conference, the organizers have established an excellent meeting series which is unique in Germany and its German-speaking neighboring countries Austria and Switzerland. This year's congress focused on the paradigm shift from the clinical use of traditional prognostic factors, such as age, tumor size and grade, to the introduction of more individualized breast cancer therapies. Conclusively, with the profound knowledge of inter- and intra-individual tumor heterogeneity on the molecular genetic level, we are able to offer subgroup-targeted therapies and more and more personalized treatments for patients with early and metastatic breast cancer.

By combining the conference with the AGO TraFo presymposium, the chairpersons created a unique platform for scientists, clinicians and young researchers interested in translational research. Therefore, we are looking forward to accept the invitation of Achim Rody and his co-chairs Michael Gnant and Nadia Harbeck to the 6th COMBATing conference in Lübeck on November 14-16, 2013.

More information about the meeting at www.combating.de 